\title{
Retrospective Analysis of Flap Based Management in Deep Sternal Wound Infection in a Tertiary Care Centre -A Single Centre Study
}

Sartaj Ahmad Guroo ${ }^{1}$, Ajit K.Padhy², Khuswant Popli², Ridhika Munjal ${ }^{1}$, Navnita Kisko ${ }^{3}$, Abhinav Gupta ${ }^{4}$

1. Resident, Department Of Cardiac Surgery, Vardhaman Mahavir Medical College and Safdarjung Hospital, New Delhi

Associate Professor, Department Of Cardiac Surgery, Vardhaman Mahavir Medical College and Safdarjung Hospital, New Delhi

3. Assistant Professor, Department Of Cardiac Surgery, Vardhaman Mahavir Medical College and Safdarjung Hospital, New Delhi

4. Professor and Head of Department, Department Of Cardiac Surgery, Vardhaman Mahavir Medical College and Safdarjung Hospital, New Delhi

Date of receiving : 5/1/2021

Date of Peer review : 23/3/2021

Date of acceptance :25/4/2021

DOI:10.47799/pimr.0902.15

\section{ABSTRACT}

Aims: In this retrospective study we analyzed the outcomes of flap based management in deep sternal wound infection (DSWI).

Materials \& Methods: Patients, who had undergone open heart surgery through median sternotomy between September 2017 and March 2020 and had developed deep sternal infections, were retrospectively analyzed in this study. Few patients found to have DSWI were managed only by Negative Pressure Wound Therapy (NPWT) and few were managed by NPWT and Bipectoral musculo fascial flap cover. The outcomes in terms of mortality and readmission in the postoperative course were obtained from the records during subsequent follow ups in OPD for six months.

Results: Out of 925 patients 11 patients (1.2\%) had deep sterna wound infection There were six patients $(n=6,54.55 \%)$ who received NPWT where as five patients $(n=5,45.45 \%)$ received flap surgery following NPWT. The patient who underwent Flap surgery had a longer postoperative stay than NPWT group (46.2+/- 22.21, C.I 95\%) days Vs (25.5+/- 14.41, C.I 95\%) days. However, the readmission due to recurrence of infection was seen only in NPWT group $(n=3,50 \%)$ with in the period of six months following discharge. One patient out of the three readmitted patients expired due to sepsis.
Conclusion: NPWT followed by bipectoral muscle flap closure has a better surgical outcome than NPW alone in deep sternal wound infection in early postoperative period

\section{INTRODUCTION}

Cardiac surgeries are widely performed through median sternotomy. Sternal wound infection following the surgery is a well known complication. The superficial wound infections settle down after wound debridement and regular antiseptic dressings, where as the deep sternal wound infection pose a significant challenge to the surgeons as it impacts significantly on prognosis of the patient and indirectly burden the patient economically with inflated hospital cost. The spectrum of management of such wounds varies from wound debridement to flap closure. In this retrospective study we analyzed the outcomes of flap based management in deep sternal wound infection.

\section{Materials and Methods:}

Patients who had undergone open heart surgery through median sternotomy and had developed Type 2a deep sternal infections, according to Glyn Jones classification of sternal wound infection ${ }^{1}$, between September 2017 and March 2020 were retrospectively analyzed in this study As per the department protocol all the patients had received shower bath following the part preparation day prior to the surgery. On 
the day of surgery the surgical sites were painted with Povidine lodine solution. Before the incision the surgical sites were draped with iodoform impregnated adhesive plastic sheet. The skin was incised with the scalpel,and electrocautery was used for subcutaneous tissue.Sternum was opened with sternal saw. In coronary artery bypass patients electrocautery was used for harvesting left internal mammary artery.

The diagnosis of deep sternal wound infection was made according to the guidelines of the centers for disease control and prevention ${ }^{2}$. Patients who met atleast one of the following criteria were included in this study :(1) Isolation of any organism from the culture of mediastinal tissue or fluid .(2) Presence of any evidence of mediastinitis seen during operation. (3) Presence of one of the following: chest pain, sternal instability (sternal dehiscence, gap between sternal halves) or fever (more than 38 degree Celsius). (4) Presence of purulent discharge from the mediastinum, necrotized tissue or isolation of any organism from the blood culture or culture of the drainage of mediastinal area.

Few patients found to have DSWI were managed only by Negative Pressure Wound Therapy (NPWT) with a periodic review of wounds after an interval of five to seven days and few were managed by NPWT and Bipectoral musculo fascial flap cover. During the course of NPWT, the decisions to intervene for a flap surgery were jointly taken by the operating team and the team of plastic surgeons, based on the persistence of positive bacterial culture following NPWT. The outcomes in terms of mortality and readmission in the postoperative course were obtained from the records during subsequent follow ups in OPD for six months. Mortality was defined by death from any cause with in the 30 days of flap reconstruction or any interval if death was related to sternal wound infection. Readmission was defined as any admission related to the complication of NPWT or flap based surgery like partial or total flap loss, nonpurulent wound dehiscence, wound infection and wound necrosis.

\section{Results}

Out of 925 patients 11 patients (1.2\%) had deep sternal wound infection The comparison of demography and the risk factors in both the groups are discussed in Table -1.

There were six patients $(n=6,54.55 \%)$ who received NPWT where as five patients ( $n=5,45.45 \%$ ) received flap surgery following NPWT. The patient who underwent Flap surgery had a longer postoperative stay than NPWT group (46.2+/- 22.21, C.I 95\%) days Vs (25.5+/- 14.41, C.I 95\%) days. However, the readmission due to recurrence of infection was seen only in NPWT group $(n=3,50 \%)$ with in the period of six months following discharge. One patient out of the three readmitted patients expired due to sepsis.

Table 1: Characteristic of patients and the outcomes of management in deep sternal wound infection patients

\begin{tabular}{|lcc|}
\hline \multicolumn{1}{|c|}{ Characteristics } & NPWT(n=6) & Flap Surgery(n=5) \\
\hline Sex & 3 & 3 \\
Male & 3 & 2 \\
Female & 1 & 3 \\
Diabetes & 5 & 0 \\
Smoking & 1 & 1 \\
Hypertension & 3 & 1 \\
Pre op. Infection & 5 & 3 \\
BMI (> 25 Kg/m2) & 3 & 0 \\
Anaemia (Hb <10mg/dl) & 1 & 0 \\
Deranged Renal function & 0 & 0 \\
Peripheral vascular disease & 0 & 0 \\
Chronic obstructive pulmonary disease & 0 & 0 \\
Use of steroids & 0 & 0 \\
Congestive heart failure & 0 & 2 \\
Sepsis/Multi organ failure & & \\
\hline
\end{tabular}




\begin{tabular}{|lcc|}
\multicolumn{1}{|c}{ Guroo et al } & WW $\mathbf{c}$ (P) 2348-1447. pimr.org.in \\
\hline Left ventricular function(<50\%) & 2 & 0 \\
Perioperative endocarditis & 0 & 3 \\
Operative Procedure & & \\
CABG & 3 & 1 \\
Valve & 3 & 1 \\
Others & 0 & 136.2 \\
CPB time (min) & 161.3 & 91.2 \\
Cross clamp time(min) & 105.3 & 0 \\
Low cardiac Output & 1 & \\
Re-exploration & 0 & 0 \\
Duration of Hospital stay (days) & $25.5+/-14.1646 .2+/-22.21$ \\
Readmission & 2 & 0 \\
Mortalitya & 1 & \\
\hline
\end{tabular}

\section{Discussion:}

The incidence of DSWI varies from 0.4 to $5 \%$. Seventy to eighty percent of patients of the wounds grow staphylococcus aureus and streptococcus epidermidis. Gardlund B et al in their study found predominantly coagulase negative staphylococci group in the patients who had sternal dehiscence related to obesity, Chronic Obstructive pulmonary disease, whereas staphylococcus aureus in patients who had perioperative contamination 3 . In our study $1.2 \%$ of patients of 925 patients had developed DSWI and majority of our patients showed growth of Methicillin resistant staph aureus (MRSA) in the wound. In one study, Hollenbeak CS et al opined that the deep chest infections increase the length of postoperative stay, which in turn increases the cost of treatment. It had been also observed by the group that the patient who were obese, and had suffered from renal insufficiency, connective tissue disease; patient who underwent re exploration and timing of antibiotic prophylaxis more than sixty minutes before the incision, had a significant higher risk of developing deep chest infection. They mentioned a mortality rate of $22 \%$ within one year postoperatively ${ }^{4}$. Similarly Toumpoulis et al in their study on post operative mortality mentioned that the early mortality rate was not influenced by the presence of deep sternal wound, but the long term mortality was higher in the deep sternal wound infection (DSWI) group than non DSWI group. Their study established the role of diabetes in $75 \%$ of the patients and preoperative renal failure on dialysis $7.5 \%$ of patientsof DSWI. They also mentioned hemodynamic instability, use of bilateral internal mammary artery, sepsis, infective endocarditis after the surgery as the independent predictors of mediastinitis5.In our study we had eight patients out of eleven $(n=8,72 \%)$ had diabetes, and deranged renal parameters in one patient ( $n=1,9 \%$ ) justifying the role of diabetes in DSWI. T szerafin et al in a retrospective study on 62 patients found $70 \%$ of the patients of DSWI had Staphylococcus aureus and Coagulase negative staphylococci infection. During their long term follow upthey studied $38.5 \%$ of patients showed recurrence and $15.4 \%$ of patients had mortality after closed suction drainage,where as $14 \%$ patients showed recurrence and $\mathbf{1 4 . 7} \%$ patients had mortality after flap based surgery. They declared the outcomes are favourable always with closed mediastinal drainage or flap closure ${ }^{6}$. Vinayak Bapat et al in their study found $80 \%$ contraction of wound size after negative suction wound therapy, which satisfied their opinion of enhanced granulation and wound contraction responsible for wound healing. They could avoid muscle flap surgery in $64 \%$ of their patients and $28 \%$ of their patients did not require surgical reconstruction at all. Finally they concluded thatthe reduced reinfection rate was associated with vaccum assisted wound closure?

Recently, Tuo Pan et al, in their comparative analysis between vaccum assisted closure versus Bilateral pectoralis major muscle flaps for deep sternal wound infection,commented a better survival rate ( $93.9 \%$ vs $74.4 \%$ ) and lesser hospital stay in flap group during their 24 months follow up ${ }^{8}$. In another study by Eleftherios $S$ et al during their follow up for 82 months they observed recurrence of infection only in 3 patients out of 55 patients who they had subjected to bilateral pectoral musculo fascial flap and opined that The functional and aesthetic outcomes are excellent with the surgery ${ }^{9}$. Similar to the above studies, we observed a better surgical out come during our 6 month follow up in Flap based surgery group, 
Which showed no recurrence or readmission, and no mortality where as there was a $50 \%$ readmission and $9 \%$ mortality seen in NPWT group. Although our study the hospital stay of flap based surgery group was more than that of NPWT group because in one patient sternal wound changed from type $2 a$ to $2 \mathrm{~b}$ resulting in prolonged hospital stay.

\section{Conclusion}

NPWT followed by bipectoral muscle flap closure has a better surgical outcome than NPW alone in deep sternal wound infection in early postoperative periods.

\section{ACKNOWLEDGEMENTS}

The research work was carried out during the years 2018 - 2020, In the Department of Cardiothoracic and Vascular Surgery, VMMC \& SJH, New Delhi. I sincerely thank Professor Anubhav Gupta, HOD, CTVS, for his guidance in making these publications possible. His dynamic, optimistic, supportive and perseverate attitude, were helpful at every stage. I thank Dr Manju Gupta for her guidance. I thank Dr Ajit kumarPadhy, Dr Khushwant Popli and Dr Aabha Divya, for their guidance both in technical and the research aspects. I would also like to extend my sincere thanks to Dr SubrataPramanik, Dr Sankalp, Dr Sudesh \& Dr Navnita, for their valuable help during the research work. I thank my seniors, Dr. Sidharth \& Dr Chandra, for guiding me for the research work. I also extend my thanks to my juniors Dr Niranjan and Dr Ridhika who helped me whenever needed. I thank my family, for backing up through the tough times of ups and downs of my career with their emotional support Finally, I dedicate this work to the patients who believed in us.

Dr. Sartaj Ahamd Guroo

Senior Resident (Acad)

Department of CTVS

VMMC \& SJH.

\section{REFERENCES}

1. Jones G, Jurkiewicz MJ, Bostwick, et al. Management of the infected median sternotomy wound with muscle flaps. Ann Surg 1997; 225:766-78.

2. Garner JS, Jarvis WR, Emori TJ, Horan TC, Hughes JM. CDC definitions for nosocomial infections. Am J Infect Control. 1988; 16: 128-40.

3. Gardlund B, Bitkover CY, Vaage J. Postoperative mediastinitisin cardiac surgery: microbiology and pathogenesis. EurJ Cardiothorac Surg 2002; 21:825-830

4. Hollenbeak CS,Murphy DM, Koenig S, et al: The clinical and economicimpact of deep chest surgical site infections followingcoronary artery bypass graft surgery. Chest 2000; 118:397.

5. Toumpoulis IK, Anagnostopoulos CE, Derose JJ Jr., Swistel DG:The impact of deep sternal wound infection on long- term survivalafter coronary artery bypass grafting. Chest 2005; 127:464.

6. Early and long term results of treatment for infected deep sternotomy wounds T Szerafin, A Peterffy . Orv Hetil 2001,Jun 24: 142(25) 1321-6

7. Experience with vaccum assisted closure of sternal wound infection following cardiac surgery and evaluation of chronic complications associated with its use. Vinayak Bapat, Naguib El-Muttardi,Christopher Young et al J card Surg, May-Jun 2008,23(3):227-33

8. Vaccum assisted closure Vs bilateral pectoralis major muscle flaps for deep sternal wound infection. Tuo Pan, Kai Li, Fu- Dong Fan, Yong -Shun Gao, Dong-Jin Wang , J Thorac Dis. 2020 Mar,12(3) : 866-875.

9. Results of the modified Bipectoral muscle flap procedures for post sternotomy deep wound infection , Eleftherios Spartalis,Charalampos,Markakis, Demetrios Moris, Elias Lachanas, E Andreas Agathos, Anna Karakatsani,Grigorios Karagkiouzis, Antonios Athanasiou, Dimitrios Dimitroulis, Perkilis Tomos, Surg Today 2016 Apr; 46(4) : 460-5

How to cite this article : Guroo SA, Padhy Ak, Popli K, Munjal R, Kisko N, Gupta A. Retrospective Analysis of Flap Based Management in Deep Sternal Wound Infection in a Tertiary Care Centre -A Single Centre Study. Perspectives in Medical Research 2021; 9 (2):69-72

DOI:10.47799/pimr.0903.15

Sources of Support: Nil, Conflict of interest: None declared 